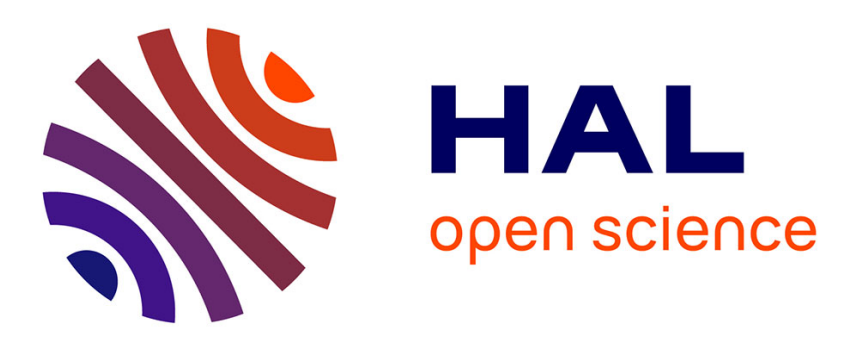

\title{
Molecular Orientational Order Probed by Coherent Anti-Stokes Raman Scattering (CARS) and Stimulated Raman Scattering (SRS) Microscopy: A Spectral Comparative Study
}

Julien Duboisset, Pascal Berto, Paulina Gasecka, Fatma-Zohra Bioud, Patrick Ferrand, Hervé Rigneault, Sophie Brasselet

\section{To cite this version:}

Julien Duboisset, Pascal Berto, Paulina Gasecka, Fatma-Zohra Bioud, Patrick Ferrand, et al.. Molecular Orientational Order Probed by Coherent Anti-Stokes Raman Scattering (CARS) and Stimulated Raman Scattering (SRS) Microscopy: A Spectral Comparative Study. Journal of Physical Chemistry B, 2015, 119 (7), pp.3242-3249. 10.1021/jp5113813 . hal-01119354

\author{
HAL Id: hal-01119354 \\ https://hal.science/hal-01119354
}

Submitted on 23 Apr 2015

HAL is a multi-disciplinary open access archive for the deposit and dissemination of scientific research documents, whether they are published or not. The documents may come from teaching and research institutions in France or abroad, or from public or private research centers.
L'archive ouverte pluridisciplinaire HAL, est destinée au dépôt et à la diffusion de documents scientifiques de niveau recherche, publiés ou non, émanant des établissements d'enseignement et de recherche français ou étrangers, des laboratoires publics ou privés. 


\title{
Molecular Orientational Order Probed by
}

\section{CoherentAnti-Stokes Raman Scattering (CARS)}

\section{and Stimulated Raman Scattering (SRS)}

\section{Microscopy: a Spectral Comparative Study}

\author{
Julien Duboisset ${ }^{\dagger},{ }^{*}$ Pascal Berto ${ }^{\dagger}$, Paulina Gasecka, Fatma-Zohra Bioud, \\ Patrick Ferrand, Hervé Rigneault, and Sophie Brasselet \\ Aix-Marseille Université, CNRS, Centrale Marseille, Institut Fresnel, UMR 7249, 13013 \\ Marseille, France \\ E-mail: julien.duboisset@fresnel.fr
}

\begin{abstract}
We investigate how to extract information on the orientational order of molecular bonds in biological samples from polarized coherent anti-Stokes Raman scattering (CARS) and stimulated Raman scattering (SRS) microscopy. Experimentally, the mean orientation of the molecular angular distribution, as well as its second and fourth orders of symmetry, are estimated by monitoring intensity signals under a varying incident polarization. We provide a generic method of analysis of polarized signals in both CARS and SRS contrasts, and apply it to imaging of lipid bonds' orientational order in multilamellar vesicles. A comparison of the two contrasts in the lipid region around $3000 \mathrm{~cm}^{-1}$ shows that while SRS allows retrieving pure molecular order information, CARS is generally tainted by a bias from the non resonant contribution.
\end{abstract}

${ }^{*}$ To whom correspondence should be addressed 


\section{Introduction}

Being able to report high level of detail in the structural molecular organization using optical microscopy is one of the prevailing goals in the field of functional imaging in biology, material sciences and molecular physics. Polarized optical microscopy is becoming a powerful tool to address molecular orientational distributions into the focal volume of a microscope. ${ }^{1}$ In coherent nonlinear optics, polarized second harmonic generation, ${ }^{2-4}$ polarized third harmonic generation ${ }^{5}$ and polarized four wave mixing ${ }^{6,7}$ have already been used to recover orientational information on endogeneous proteins and lipids in biological tissues. In addition to the orientational information, Coherent Raman Scattering (CRS) processes are sensitive to molecular bond vibrations, allowing chemical specificity without the need of fluorescence labelling. ${ }^{8-10}$

Coherent anti-Stokes Raman scattering (CARS) microscopy is based on the interaction of two fields called pump $\left(\omega_{p}\right)$ and Stokes $\left(\omega_{s}\right) .{ }^{11}$ When their frequency difference matches a vibrational resonance $\left(\Omega_{R}\right)$, a blue-shifted field called anti-Stokes $\left(\omega_{a s}=2 \omega_{p}-\omega_{s}\right)$ is generated. In this case, the intensity recovered at $\omega_{a s}$ reveals the presence of specific bonds but is quadratically dependent on the nonlinear susceptibility and can interfere with a spurious nonresonant background. ${ }^{12}$ In recent years, the implementation of another CRS process called Stimulated Raman Scattering (SRS), has become an increasingly used tool for labelfree microscopy. ${ }^{14,15}$ Simultaneously to the generation of an anti-Stokes photon, a gain on the Stokes beam (stimulated Raman gain, SRG) and a loss on the pump beam (stimulated Raman loss, SRL) appears when the frequency difference $\omega_{p}-\omega_{s}$ matches $\Omega_{R}$. To detect the SRG signal (resp. SRL signal), the pump beam (resp. Stokes beam) is modulated at high frequency, typically in the $\mathrm{MHz}$ range, and the gain on the Stokes beam (resp. the loss on the pump beam) is measured by means of a lock-in amplifier. SRS is a background free process which allows to recover directly pure molecular Raman spectra. Even though CARS and SRS techniques are sensitive to the same Raman susceptibility tensor, the information recovered by both techniques differ by their underlying physical mechanism. 
We have previously developed a generic polarization resolved microscopy technique able to retrieve molecular orientational information in organized lipid membranes, based on symmetry orders decomposition of the lipid orientational distribution function. ${ }^{7}$ The information retrieved however neglected the non resonant background intrinsic to the CARS signal because of the high contrast obtained on the considered resonance. This nonresonant contribution is known however to perturb the interpretation of polarized signals in more general cases. ${ }^{27}$ On the other hand, SRS polarized microscopy studies have been introduced so far only in isotropic media. ${ }^{6}$ A unified approach for both contrasts, encompassing the whole characteristics of both of them, has not yet been developed.

Here we propose to investigate the nature of polarization resolved CARS and SRS signals, and in particular their respective capacity to disentangle molecular orientational information in the focal volume of a microscope. We introduce an exhaustive model to analyze polarization resolved SRS signals, similarly to what has been already acheived for CARS. ${ }^{7}$ We illustrate the method on multilamellar vesicles to extract lipid orientational organization. Finally, we compare the CARS and SRS polarization resolved methods by extending the analysis to the frequency domain, addressing more specifically details in the vibrational lipid bond spectral region.

\section{Macroscopic nonlinear susceptibility}

The resonant third order nonlinear susceptibility $\chi$ of vibrational mode is built-up based on the sum of individual nonlinear dipoles, describing the molecular bonds contributing to this vibrational mode. ${ }^{5,16}$ Each of these nonlinear dipoles, defined by their direction $(\theta, \varphi)$ in the macroscopic laboratory frame, are characterized by a nonlinear susceptibility $\gamma$ in the microscopic frame. In the case of $\mathrm{CH}_{2}$ vibrational bonds studied below, only the $\gamma_{z z z z}$ is assumed non-zero.$^{5}$ For more complex vibrational bonds, all the components of the susceptibility tensor have to be taken into account. ${ }^{16}$ In the laboratory frame, the probability 
a)

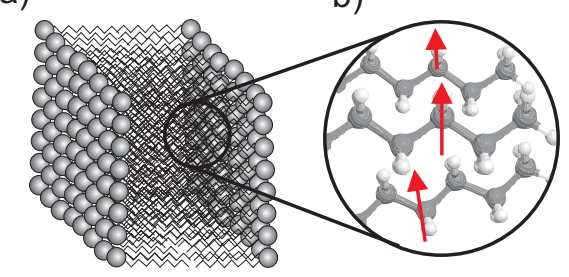

c)

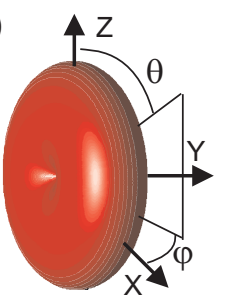

d)

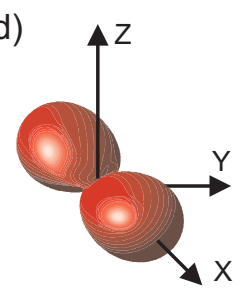

e)

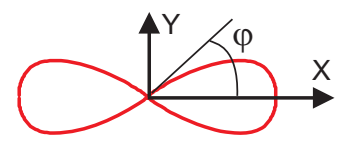

f)

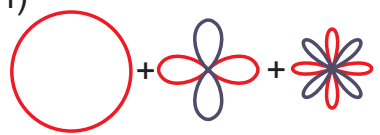

Figure 1: a) Assemblies of lipids into the focal volume. b) Orientation of nonlinear induced dipoles, assumed to lie along the $C-H$ bonds probed, defined in the laboratory frame $X Y Z$ c) 3D nonlinear dipoles distribution defined into the focal volume $f(\theta, \varphi)$. d) 3D nonlinear dipoles distribution which contributes to the signal. e) $\tilde{p}(\varphi)$ distribution function. f) Decomposition of the distribution function $\tilde{p}(\varphi)$ on the spherical function basis. (Color online)

to find individual nonlinear dipoles with an orientation $\theta$ and $\varphi$ is defined by a function $f(\theta, \varphi)$. The macroscopic nonlinear susceptibility tensor in the laboratory frame is defined as the summation over individual dipoles probed in the focal volume

$$
\chi_{I J K L}=N \int_{0}^{\pi} \int_{0}^{2 \pi} f(\theta, \varphi)(I . z)(J . z)(K . z)(L . z) \gamma \sin \theta \mathrm{d} \theta \mathrm{d} \varphi,
$$

where $N$ is the molecular bonds density, $(I . z)$ is the projection of the direction $I$ along $z, \gamma$ is the microscopic tensor of one elementary molecular bond, see Fig. 1.

Due to the electromagnetic fields which are lying in the XY sample plane, only the $X$ and $Y$ components of the macroscopic tensor are probed. The out-of plane contributions of the collection of dipoles in the focal volume will consequently contribute far less than the in-plane ones in the $\theta$ orientational average. From Eq. 1, the in-plane components of the macroscopic tensor are

$$
\begin{aligned}
& \chi_{X X X X}=N \gamma \int_{0}^{2 \pi} p(\varphi) \cos ^{4}(\varphi) \mathrm{d} \varphi \\
& \chi_{Y Y Y Y}=N \gamma \int_{0}^{2 \pi} p(\varphi) \sin ^{4}(\varphi) \mathrm{d} \varphi \\
& \chi_{X X Y Y}=N \gamma \int_{0}^{2 \pi} p(\varphi) \cos ^{2}(\varphi) \sin ^{2}(\varphi) \mathrm{d} \varphi \\
& \chi_{X Y Y Y}=N \gamma \int_{0}^{2 \pi} p(\varphi) \cos (\varphi) \sin ^{3}(\varphi) \mathrm{d} \varphi \\
& \chi_{Y X X X}=N \gamma \int_{0}^{2 \pi} p(\varphi) \cos ^{3}(\varphi) \sin (\varphi) \mathrm{d} \varphi
\end{aligned}
$$


with

$$
p(\varphi)=\int_{0}^{\pi} \sin ^{4} \theta f(\varphi, \theta) \sin \theta \mathrm{d} \theta
$$

where the term $\sin ^{4}(\theta)$ weights the contribution of molecular bonds depending on their tilt angle $\theta$. Without loss of generality, the molecular bonds distribution in the $X Y$ plane can be described by a function of $\varphi$. Moreover, Eq. 2 clearly show that the tensor components are sensitive to the even part of the $p(\varphi)$ function. Furthermore, decomposing $p(\varphi)$ onto its orders of symmetry leads to only three non vanishing contributions of this distribution function to the third order susceptibility: its isotropic contribution as well as its second and fourth orders. Thereby, the 2D distribution function of Eq. 3 can be expanded as a truncated Fourier series limited to even order terms, see Fig. 1e-f, and modeled by ${ }^{7}$

$$
\begin{aligned}
\tilde{p}(\varphi)= & p_{0}+p_{2} \cos (2 \varphi)+q_{2} \sin (2 \varphi) \\
& +p_{4} \cos (4 \varphi)+q_{4} \sin (4 \varphi) .
\end{aligned}
$$

Then, from Eq 2 and 4, the macroscopic susceptibility components in the $O X Y$ plane can be written with respect to the coefficients of the distribution function as

$$
\begin{aligned}
& \chi_{X X X X}=\frac{N \gamma \pi}{8}\left(6 p_{0}+4 p_{2}+p_{4}\right) \\
& \chi_{Y Y Y Y}=\frac{N \gamma \pi}{8}\left(6 p_{0}-4 p_{2}+p_{4}\right) \\
& \chi_{X X Y Y}=\frac{N \gamma \pi}{8}\left(2 p_{0}-p_{4}\right) \\
& \chi_{X Y Y Y}=\frac{N \gamma \pi}{8}\left(2 q_{2}-q_{4}\right) \\
& \chi_{Y X X X}=\frac{N \gamma \pi}{8}\left(2 q_{2}+q_{4}\right) .
\end{aligned}
$$

The in-plane tensor components are clearly dependent on the matter organization described by the five distribution function parameters $p_{0}, p_{2}, q_{2}, p_{4}$ and $q_{4}$. Henceforth, the CARS and SRS intensities have to be measured using different sets of polarization to recover these parameters, as described in the next section. 


\section{Polarization-resolved SRS intensity}

The stimulated Raman gain (SRG) process is based on the interference between the electric field induced by the nonlinear dipole at $\omega_{s}$ and the incoming field at $\omega_{s} .{ }^{13,14}$ Due to this heterodyne behavior, the gain on the Stokes beam at resonance is linear with the susceptibility, and reads ${ }^{17}$

$$
I^{S R G} \propto \sum_{I J K L} \chi_{I J K L} E_{p, I}^{*} E_{s, J} E_{p, K} E_{s, L}^{*}
$$

where $E_{p}$ denotes the pump electric field, $E_{s}$ the Stokes electric field, and $\chi$ the resonant susceptibility tensor as previously discussed. Both incident fields $E_{p}$ and $E_{s}$ are linearly polarized. Denoting $\alpha$ the angle of $E_{s}$ and $E_{p}$ with respect to the $X$ axis (see inset Fig 2), the electric fields can be expressed in the macroscopic frame as $E_{X}=E \cos \alpha$ and $E_{Y}=E \sin \alpha$. Then Eq 6 becomes

$$
\begin{aligned}
I^{S R G}(\alpha) \propto & \chi_{X X X X} \cos ^{4} \alpha+4 \chi_{X X X Y} \cos ^{3} \alpha \sin \alpha \\
& +6 \chi_{X X Y Y} \cos ^{2} \alpha \sin ^{2} \alpha \\
& +4 \chi_{X Y Y Y} \cos \alpha \sin ^{3} \alpha+\chi_{Y Y Y Y} \sin ^{4} \alpha .
\end{aligned}
$$

This equation can be rearranged as

$$
\begin{aligned}
I^{S R G}(\alpha) \propto a_{0} \quad & +a_{2} \cos (2 \alpha)+b_{2} \sin (2 \alpha) \\
& +a_{4} \cos (4 \alpha)+b_{4} \sin (4 \alpha),
\end{aligned}
$$

where

$$
\begin{aligned}
a_{0}=\frac{3 \pi}{4} p_{0} \quad a_{2} & =\frac{\pi}{2} p_{2} \quad a_{4}=\frac{\pi}{8} p_{4} \\
b_{2}=\frac{\pi}{2} q_{2} & b_{4}=\frac{\pi}{8} q_{4} .
\end{aligned}
$$

The polarization-resolved SRS intensity is thus described as a truncated Fourier series where the Fourier coefficients are linearly related to the distribution function parameters. This way, the polarization-resolved SRS signal allows one to retrieve directly how the unitary dipoles, 
representing the molecular bonds, are oriented and distributed into the focal volume.

\section{Polarization-resolved CARS Intensity}

\section{Exact calculation}

The CARS dipole along the direction $I(X$ or $Y)$, expressed as a function of the nonlinear susceptibility and the electric fields, is

$$
P_{I}\left(\omega_{a s}\right)=\sum_{J K L} \chi_{I J K L} E_{s, J}^{*} E_{p, K} E_{p, L}
$$

The CARS intensity behind a polarizer oriented along $X$ is thus proportional to the square of the CARS dipole which leads to

$$
I_{X}^{C A R S} \propto \sum_{\substack{J K L \\ M N O}} \chi_{X J K L} \chi_{X M N O}^{*} E_{J}^{*} E_{K} E_{L} E_{M} E_{N}^{*} E_{O}^{*}
$$

The total intensity is thus the summation of the intensity along $X$ plus the intensity along $Y$

$$
I^{C A R S}=I_{X}^{C A R S}+I_{Y}^{C A R S}
$$

Both incident $E_{p}$ and $E_{s}$ are linearly polarized and rotated in the $O X Y$ plane in the polarization resolved SRS operating mode. $I^{C A R S}$ is rewritten as a truncated Fourier series where the angular frequencies are going up to the sixth order due to the presence of six electric fields in the intensity relationship. ${ }^{7,18}$ In this case,

$$
\begin{aligned}
I^{C A R S}(\alpha) \propto a_{0} \quad & +a_{2} \cos (2 \alpha)+b_{2} \sin (2 \alpha) \\
& +a_{4} \cos (4 \alpha)+b_{4} \sin (4 \alpha) \\
& +a_{6} \cos (6 \alpha)+b_{6} \sin (6 \alpha)
\end{aligned}
$$


where

$$
\begin{aligned}
& a_{0}=\frac{1}{64}\left(36 p_{0}^{2}+10 p_{2}^{2}+10 q_{2}^{2}+p_{4}^{2}+q_{4}^{2}\right) \\
& a_{2}=\frac{1}{32}\left(24 p_{0} p_{2}+3 p_{2} p_{4}+3 q_{2} q_{4}\right) \\
& a_{4}=\frac{3}{32}\left(p_{2}^{2}-q_{2}^{2}+2 p_{0} p_{4}\right) \\
& a_{6}=\frac{1}{32}\left(p_{2} p_{4}-q_{2} q_{4}\right) \\
& b_{2}=\frac{1}{32}\left(p_{0} q_{2}+3 p_{2} q_{4}-3 p_{4} q_{2}\right) \\
& b_{4}=\frac{3}{16}\left(2 p_{0} q_{4}+p_{2} q_{2}\right) \\
& b_{6}=\frac{1}{32}\left(p_{2} q_{4}+p_{4} q_{2}\right)
\end{aligned}
$$

In the polarization-resolved CARS signal, the retrieval of the distribution function from the Fourier coefficients is therefore not straightforward. The nonlinear dependence of the Fourier coefficients with respect to the distribution function parameters is due to the nonlinear dependence of the CARS signal with respect to the susceptibility tensor, which is not the case in SRS. To estimate the distribution function parameters, an iterative algorithm can be employed to find the right set of parameters. ${ }^{7}$

\section{Approximate calculation}

The nonlinear dependence between the polarization-resolved CARS intensity and the distribution function leads in practice to a complex and time consuming data processing. Nevertheless, an approximation can be done in order to simplify and decrease the time of analysis. As the CARS intensity is quadratically dependent on the susceptibility, it can be written as the square of a truncated Fourier series

$$
\begin{aligned}
\tilde{I}^{C A R S}(\alpha) \propto \quad & {\left[a_{0}^{\prime}+a_{2}^{\prime} \cos (2 \alpha)+b_{2}^{\prime} \sin (2 \alpha)\right.} \\
& \left.+a_{4}^{\prime} \cos (4 \alpha)+b_{4}^{\prime} \sin (4 \alpha)\right]^{2}
\end{aligned}
$$


When Eq. 15 is developed, the parameters $a_{0}, a_{2}, a_{4}, b_{2}$ and $b_{4}$ can be expressed as functions of $a_{0}^{\prime}, a_{2}^{\prime}, a_{4}^{\prime}, b_{2}^{\prime}$ and $b_{4}^{\prime}$,

$$
\begin{aligned}
& a_{0}=a_{0}^{\prime 2}+a_{2}^{\prime 2} / 2+b_{2}^{\prime 2} / 2+a_{4}^{\prime 2}+b_{4}^{\prime 2} \\
& a_{2}=2 a_{0}^{\prime} a_{2}^{\prime}+a_{2}^{\prime} a_{4}^{\prime}+b_{2}^{\prime} b_{4}^{\prime} \\
& a_{4}=a_{2}^{\prime 2} / 2-b_{2}^{\prime 2} / 2+2 a_{0}^{\prime} a_{4}^{\prime} \\
& a_{6}=a_{2}^{\prime} a_{4}^{\prime}-b_{2}^{\prime} b_{4}^{\prime} \\
& b_{2}=2 a_{0}^{\prime} b_{2}^{\prime}+a_{2}^{\prime} b_{4}^{\prime}-a_{4}^{\prime} b_{2}^{\prime} \\
& b_{4}=2 a_{0}^{\prime} b_{4}^{\prime}+a_{2}^{\prime} b_{2}^{\prime} \\
& b_{6}=a_{2}^{\prime} b_{4}^{\prime}+a_{4}^{\prime} b_{2}^{\prime} .
\end{aligned}
$$

Eq. 16 shows a clear similarity to Eq. 14. From this, an approximate relationship can be found between the parameters $a_{n}^{\prime}, b_{n}^{\prime}$ and the parameters $p_{n}, q_{n}$,

$$
\begin{aligned}
a_{0}^{\prime} \approx \frac{3 \pi}{4} p_{0} \quad a_{2}^{\prime} \approx \frac{\pi}{2} p_{2} \quad a_{4}^{\prime} \approx \frac{\pi}{8} p_{4} \\
b_{2}^{\prime} \approx \frac{\pi}{2} q_{2} \quad b_{4}^{\prime} \approx \frac{\pi}{8} q_{4} .
\end{aligned}
$$

In a first approximation, the square root of the polarization-resolved CARS signal is very similar the polarization-resolved SRS signal. A numerical comparison was done between the exact and the approximate expressions of the CARS signal, and the two outcomes were seen to be identical within $1 \%$ whatever the set of $a_{n}, b_{n}$ parameters considered. The negligible difference comes from the eighth frequency present in $\tilde{I}$, whereas $I$ contains up to the 6 th order frequency in its $\alpha$ dependence. In what follows, the data analysis uses the approximate calculation. 


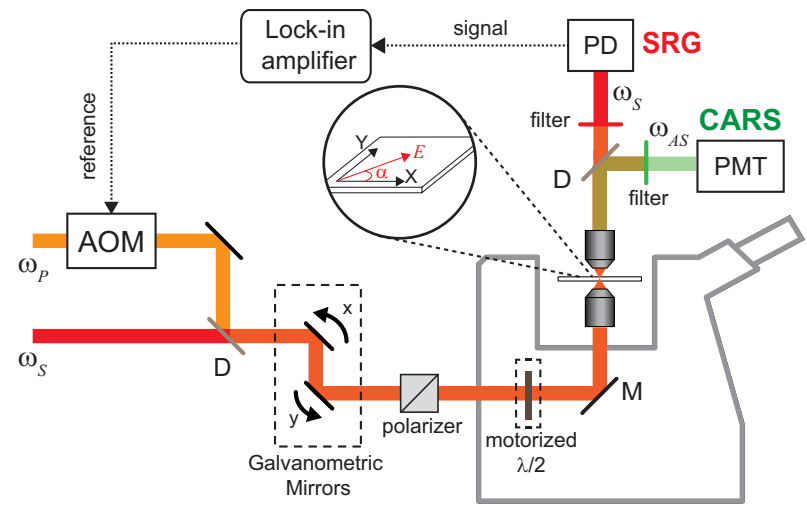

Figure 2: Description of the polarization-resolved CRS microscope setup. The linear polarization orientation of both exciting beams is controlled by a motorized achromatic half wave plate placed before the excitation objective. AOM: Acousto-optic modulator, PD: Photodiode, PMT: Photomultiplier tube, D: Dichroic mirror, M: Metallic mirror. (Color online)

\section{Data processing}

CARS and SRS polarimetric stacks are obtained by measuring a sequence of CARS and SRS images for different polarization angles $\alpha$ between 0 and $175^{\circ}$ with a $5^{\circ}$ step. Consequently, $\sqrt{I^{C A R S}(\alpha)}$ and $I^{S R S}(\alpha)$ can be easily extracted on each pixel. According to Eq. 9 related to SRS and Eq. 17 related to CARS, the distribution function parameters $p_{0}, p_{2}, q_{2}, p_{4}, q_{4}$ are determined on each pixel. From the distribution function parameters, four parameters can be introduced to quantify the symmetry order magnitude and the main orientation of the distribution function. First, we introduce $S_{2}$ and $S_{4}$ to quantify the order of symmetry regardless of the orientation of the molecular bonds ${ }^{7}$

$$
\begin{aligned}
& S_{2}=\frac{1}{p_{0}} \sqrt{p_{2}^{2}+q_{2}^{2}} \\
& S_{4}=\frac{1}{p_{0}} \sqrt{p_{4}^{2}+q_{4}^{2}} .
\end{aligned}
$$


Moreover, we introduce the angles $\varphi_{2}$ and $\varphi_{4}$ to describe respectively the order 2 and the order 4 orientations of the distribution function in the sample plane

$$
\begin{aligned}
& \varphi_{2}= \begin{cases}\frac{1}{2} \arctan \left(q_{2} / p_{2}\right), & \text { if } p_{2}>0 \\
\frac{1}{2} \arctan \left(q_{2} / p_{2}\right)+\frac{\pi}{2}, & \text { else }\end{cases} \\
& \varphi_{4}= \begin{cases}\frac{1}{4} \arctan \left(q_{4} / p_{4}\right), & \text { if } p_{4}>0 \\
\frac{1}{4} \arctan \left(q_{4} / p_{4}\right)+\frac{\pi}{4}, & \text { else }\end{cases}
\end{aligned}
$$

These four independent parameters play distinct roles in the description of the function distribution. The angle $\varphi_{2}$ reports on the main orientation of the distribution function. This can be emphasized by writing all angular dependences relative to $\varphi_{2},{ }^{19}$

$$
\begin{aligned}
S_{4}^{(s)} & =S_{4} \cos \left(4\left(\varphi_{4}-\varphi_{2}\right)\right) \\
S_{4}^{(a)} & =S_{4} \sin \left(4\left(\varphi_{4}-\varphi_{2}\right)\right) .
\end{aligned}
$$

$S_{4}^{(s)}$ describes the magnitude of the "symmetric" part of the order 4 contribution to the distribution function $p(\varphi)$, meaning that the symmetric order four is either aligned or at $\pi / 4$ with $\varphi_{2}$. The $S_{4}^{(a)}$ parameter is the "antisymmetric" order four, reporting a skewness in the distribution function. ${ }^{19}$

The SRS and CARS polarization-resolved intensities are finally expressed as

$$
\left.\sqrt{\sqrt{I^{C A R S}(\alpha)}}\right\} \begin{aligned}
& \\
& \\
& \\
& +\frac{1}{6} S_{4}^{(s)} \cos \left(4\left(\alpha-\varphi_{2}\right)\right) \\
& +\frac{1}{6} S_{4}^{(a)} \sin \left(4\left(\alpha-\varphi_{2}\right)\right),
\end{aligned}
$$

where it is visible that a simple Fourier analysis of the polar-resolved intensities allows one 
to retrieve the distribution function parameters $S_{2}, S_{4}^{(s)}, S_{4}^{(a s)}$ and $\varphi_{2}$.

\section{Experiments}

Multilamellar vesicles (MLVs) were made from DPPC lipids (1,2-dipalmitoyl-sn-glycero-3phosphocholine). Thin films, made of pure DPPC were hydrated in phosphate-buffered saline (PBS, pH 7.4) for 1 hour above the main phase transition temperature $\left(45^{\circ} \mathrm{C}\right)$ leading to MLVs of 1-30 $\mu \mathrm{m}$ size according to the procedure described in the literature. ${ }^{20}$

The experimental setup is sketched in Fig. 2. A modelocked Nd:YVO4 MOPA laser (Plecter, Coherent, $80 \mathrm{MHz}$ repetition rate) delivers synchronous 7 ps pulses at $\lambda_{S}=1064 \mathrm{~nm}$ (used as Stokes beam) and $\lambda=532 \mathrm{~nm}$. This frequency doubled beam is used to pump an OPO (APE Levante OPO) which generates the tunable pump/probe pulse with a maximal tuning range of $\lambda_{P}=730-960 \mathrm{~nm}$. The two beams are overlapped in time and space and sent into a custom made scanning microscope. ${ }^{21,23}$ In the CARS operation mode the anti-Stokes signal is filtered and detected, in the forward direction, with an photomultiplier tube (R9110, Hamamatsu) coupled with a preamplifier (C7950, Hamamatsu). In the SRS operation mode, the pump beam is modulated in amplitude at the frequency of $20 \mathrm{MHz}$ thanks to an acoustooptic modulator (AOM - AAOptoelectronic MT200 A0,2-800). The stimulated Raman gain (SRG) induced on the Stokes beam is detected in the forward direction by means of a

high speed photodiode and a fast lock-in amplifier manufactured by APE. ${ }^{15}$ The collection objective lens (NA=0.6, LUCPlanFLN 40x, Olympus) has a larger numerical aperture than the excitation one (NA=0.45, LCLPLN 20x IR, Olympus) to minimize artifacts induced by cross phase modulation and thermal lensing. ${ }^{22}$ For CARS and SRS measurements, the power of the Stokes and pump beams in the sample plane was set respectively to $28 \mathrm{~mW}$ and 39.5 $\mathrm{mW}$. Each spectral image results from an average of 3 images, using a pixel dwell time of $100 \mu s$. To perform polarization resolved measurements, both exciting beams are linearly polarized before the excitation objective, and an achromatic half-waveplate mounted in a 
step rotation motor allows rotation of the incident pump and Stokes polarizations at the same time. In this configuration, polarization distortions introduced by the experimental setup, such as ellipticity, were measured as negligible.
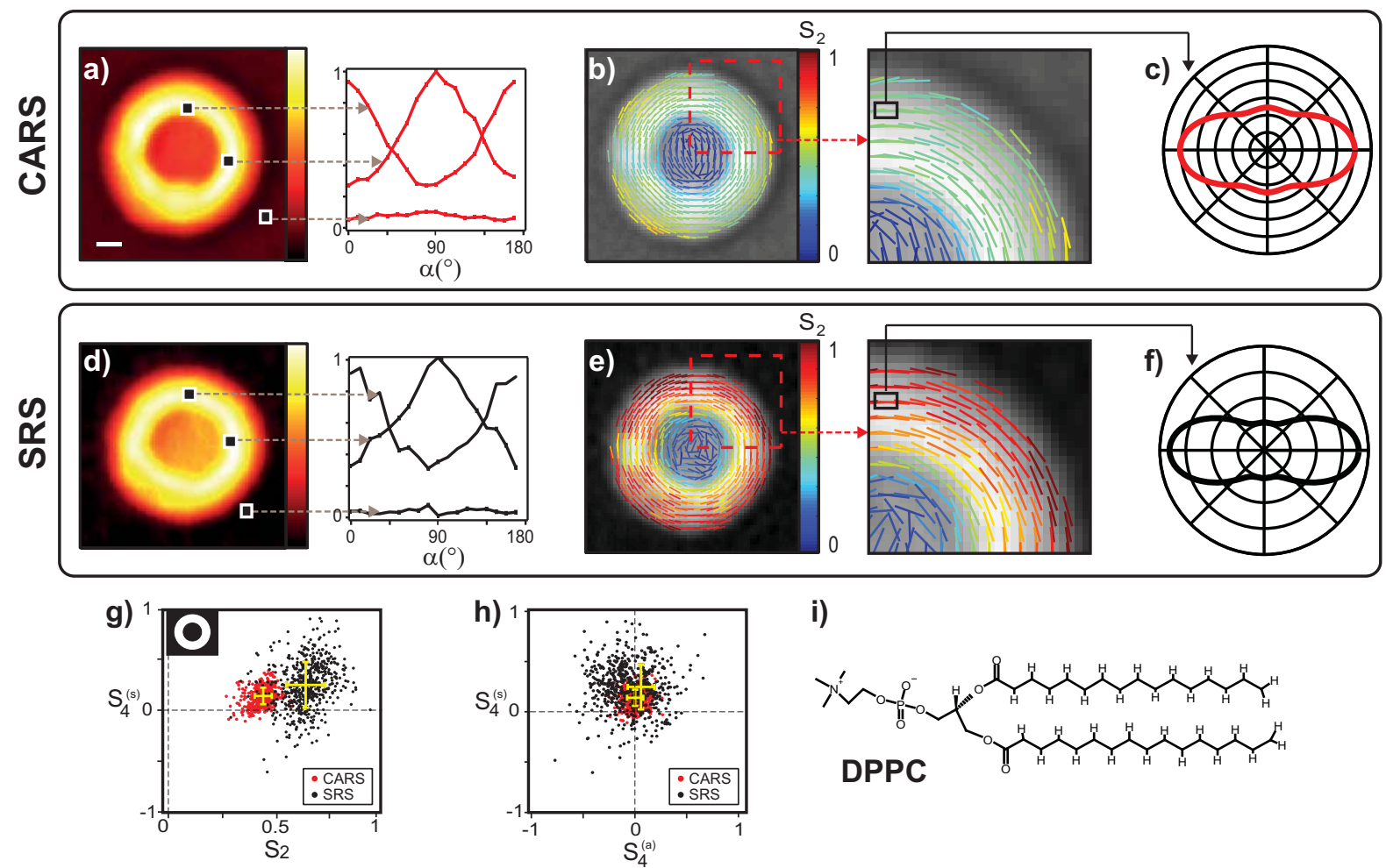

i)

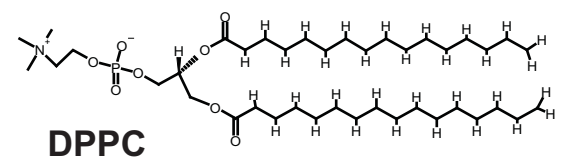

Figure 3: (a) Total CARS intensity at $2836 \mathrm{~cm}^{-1}$ and polarization resolved signal in different regions of interest in the $\mathrm{MLV}$. The scale bar represents $10 \mu \mathrm{m}$. (b) The stick orientation represents $\varphi_{2}$ and the color indicates the value of $S_{2}$. (c) Distribution function $\tilde{p}(\varphi)$ for a given pixel. (d-f) Total SRS intensity at $2850 \mathrm{~cm}^{-1}$ and identical analysis as for CARS. (g-h) Plot of $S_{2}$ and $S_{4}$ values (in red for CARS, in black for SRS) for each pixel of the image within a given mask (inset Fig 3g) applied to remove all the pixels from the background. The center of the yellow cross represents the mean, and the error bar represents the standard deviation of the measured values. (i) Schematic representation of a DPPC molecule. (Color online) 


\section{Results}

\section{Polarization-resolved CARS and SRS intensities on multilamellar vesicles}

The experimental results are depicted in Fig. 3. The MLVs form spherical objects made by several concentric layers where the DPPC lipids, see Fig. 3i, are radially oriented whereas the $\mathrm{CH}_{2}$ bonds are tangentially oriented. ${ }^{7,26}$ CARS and SRS polarization resolved image stacks were recorded on the same MLV, at the $\mathrm{CH}_{2}$ stretching mode resonance. Figure $3 a, d$ is the summation over the whole stack and shows a strong signal from the equatorial plane of the MLV. The background in the CARS image is non zero and comes from non resonant contribution of the surrounding water whereas the SRS image is background free. The polarization-resolved intensities in the MLV are strongly modulated as function of the incident polarization angle $\alpha$ as being the signature of oriented nonlinear induced dipoles, whereas the surrounding background shows no polarization dependence, as expected from an isotropic medium.

From the analysis of the polarization-resolved intensities previously discussed, the distribution function parameters are measured on each pixel (Eq 23). Superimposed to the black and white intensity image, a stick is added on each pixel whose orientation is given by $\varphi_{2}$ and whose color follows the $S_{2}$ value scale (Fig. $4 \mathrm{~b}, \mathrm{e}$ ). The $\varphi_{2}$ orientation within the MLV shows that the $\mathrm{CH}_{2}$ bonds are found to be oriented tangentially with respect to the MLV circular contour, as expected from the lipids geometry in a concentric arrangement and the arrangement of the $\mathrm{CH}_{2}$ symmetric stretching bonds along the lipid chains. The $S_{2}$ values reveal a strong organization in the equatorial plane. In the center of the MLV, $S_{2}$ is close to zero as expected for random lipids orientation. The $S_{4}$ values are much weaker revealing a function distribution mainly unidirectional. To visualize the statistical extent of the measured distribution function parameters over the oriented region of the MLV section, a mask has been applied to the CARS and SRS images, keeping all intensity contributions 
well above the background (see inset Fig. 3g). For each remaining pixel of the image, $S_{4}^{(s)}$ as function of $S_{2}$ and $S_{4}^{(a)}$ are reported in a $2 \mathrm{D}$ graph as represented Fig. 3g-h. The distribution of observed data points is due to the heterogeneity in the MLV and the noise of the measurement. Figure $3 \mathrm{~g}-\mathrm{h}$ shows first that the $S_{4}^{(a)}$ values obtained from both CARS and SRS are centered on zero, which is a signature of angular distributions of cylindrical symmetry, as expected from the arrangement of the $C-H$ molecular bonds in the MLV geometry. More strikingly, a clear difference can be observed in the $S_{2}$ and $S_{4}^{(s)}$ coefficients obtained from CARS and SRS analyses. Using the obtained symmetry parameters and Eq. 4, it is possible to represent the reduced distribution function $\tilde{p}(\varphi)$ read-out by polarization resolved CARS and SRS. This distribution, represented in Fig. 3c-f, shows an obvious anisotropic shape along the $\mathrm{CH}_{2}$ vibration bond axes, with minor fourth order contributions, especially in the SRS analysis. In order to explain this difference, a finer analysis of the nature of these processes is required, in particular a spectral investigation was performed around the lipid resonance, as described in the next section.

\section{Spectral behavior of CARS and SRS}

A polarization resolved CARS and SRS analysis has been performed in the whole $2750 \mathrm{~cm}^{-1}$ to $3000 \mathrm{~cm}^{-1}$ spectral region of the lipid vibrational resonance, in the same MLV equatorial plane as described in the previous section. The total intensity depicted in Fig. 4a shows a strong signal around the lipid resonance. Three strong CARS and SRS features are distinguishable: the two bands around $2848 \mathrm{~cm}^{-1}$ and $2880 \mathrm{~cm}^{-1}$ correspond to the symmetric and asymmetric $\mathrm{CH}_{2}$ stretching vibrations, whereas the band at $2935 \mathrm{~cm}^{-1}$ corresponds to the symmetric $\mathrm{CH}_{3}$ stretching.${ }^{24}$ Fig 4 a shows a typical CARS spectrum representative of a coherent superposition of a spectrally-independent non resonant signal, and a complex spectral resonance. In contrast, the SRS spectrum reproduces directly the shape of a lipid Raman spectrum. Moreover, the SRS spectrum is clearly background-free out of resonance, whereas the CARS spectrum shows a non resonant background of around $30 \%$ of the maxi- 


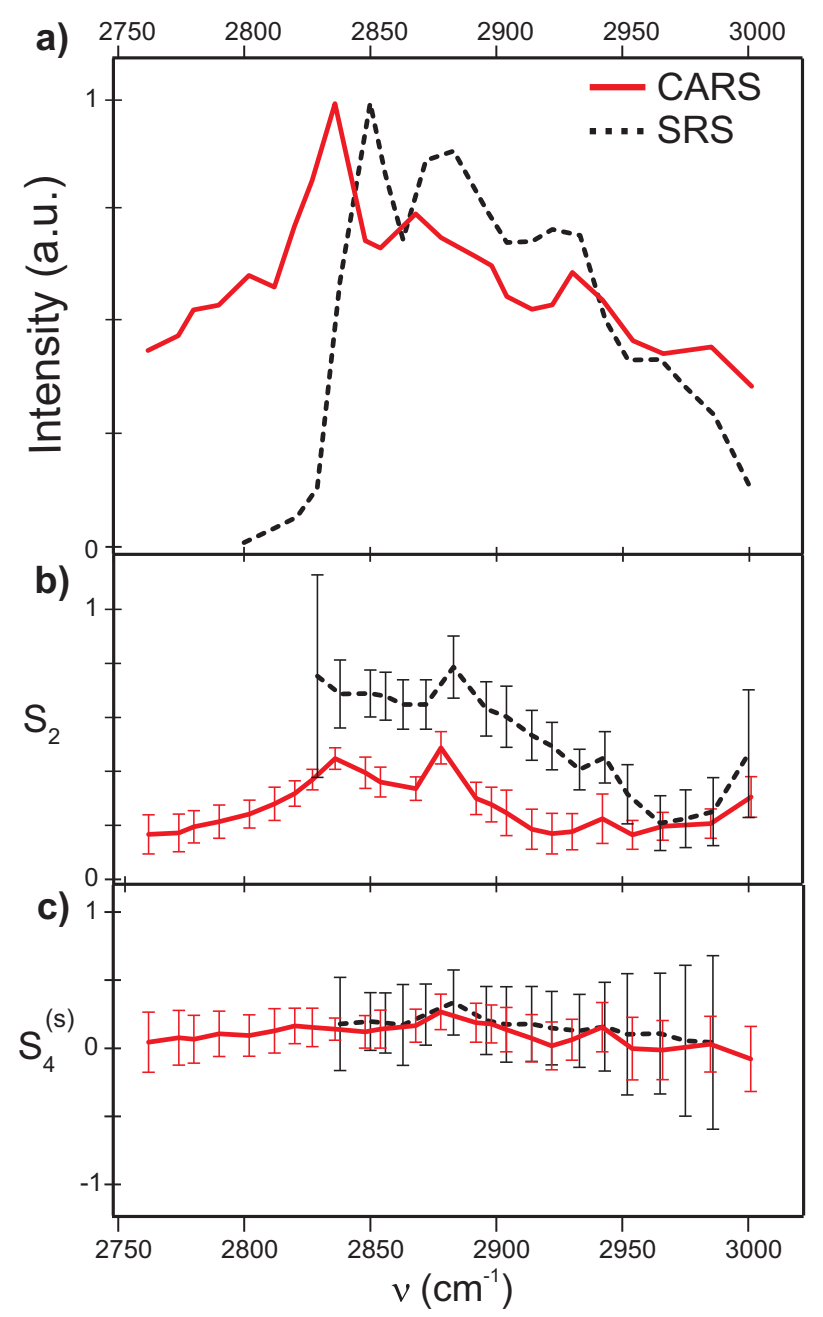

Figure 4: a) spectra of the MLV. b) $S_{2}$ as a function of the frequency for CARS (line) and SRS (dashed line). c) $S_{4}^{(s)}$ as a function of the frequency for CARS (line) and SRS (dashed line). (Color online)

mum intensity.

Fig. 4b shows the $S_{2}$ spectral dependence, obtained from a polarization analysis at each addressed wavenumber, for both CARS and SRS. The reported values are obtained from the average and standard deviation over a collection of pixels within the mask of interest depicted in Fig. 3g. The values of $S_{2}$ for the CARS starts from a low value out of resonance to reach two maxima around the symmetric and asymmetric $\mathrm{CH}_{2}$ stretching $\left(S_{2} \approx 0.4\right)$. The $S_{2}$ values for the symmetric and asymmetric stretching are the same because the $\mathrm{CH}_{2}$ angular distribution is identical for the two frequencies. Indeed while the microscopic nature of the 
vibrational bond symmetry might be different, the dominant effect in the obtained symmetry orders is the overall angular extent of the individual dipoles constituting these vibrational bond, represented here by the $\mathrm{CH}_{2}$ directions. The decrease of $S_{2}$ in the intermediate spectral region between these two resonances is more difficult to interpret, mainly because the overlapped contribution of the neighbouring spectral regions become non negligible. The $S_{2}$ value reached at the $\mathrm{CH}_{3}$ stretching mode is obviously lower than for the $\mathrm{CH} 2$ stretching modes. The $\mathrm{CH}_{3}$ group, present at the head and the tail of the lipid is simply less oriented in the MLV, most probably because of its lowered angular constraint at the extremity of the lipid leaflets, see Fig. 3i. The values of $S_{2}$ for SRS show the same behavior with high values $\left(S_{2} \approx 0.7\right)$ at the $\mathrm{CH}_{2}$ stretching resonances (the analysis has been restricted to a frequency domain where the SRS signal was measurable). Similarly as for CARS, the $\mathrm{CH}_{3}$ vibrational bond region shows a lower $S_{2}$ value.

Figure 4c represents the $S_{4}^{(s)}$ values for SRS and CARS as functions of the wavenumber. We observe that the $S_{4}^{(s)}$ values do not surpass 0.25 , showing the absence of high order in the distribution function as reported in Ref. ${ }^{7}$

\section{Discussion}

Even though the CARS and SRS processes are different, both techniques are expected to lead to the same molecular orientational distribution. However, as seen in Fig. 3 and 4, a clear difference is visible on the $S_{2}$ values measured in MLV, which are seen to be systematically higher in the SRS operating mode. Indeed, the assumption made in the theoretical part related to the CARS operating mode does not take into account the other contributions from the non resonant part. Indeed, the CARS intensity spectrum shows a non resonant contribution that can disturb the interpretation of the values obtained by the polarizationresolved technique.

In order to understand the difference observed with SRS and CARS, a model was de- 


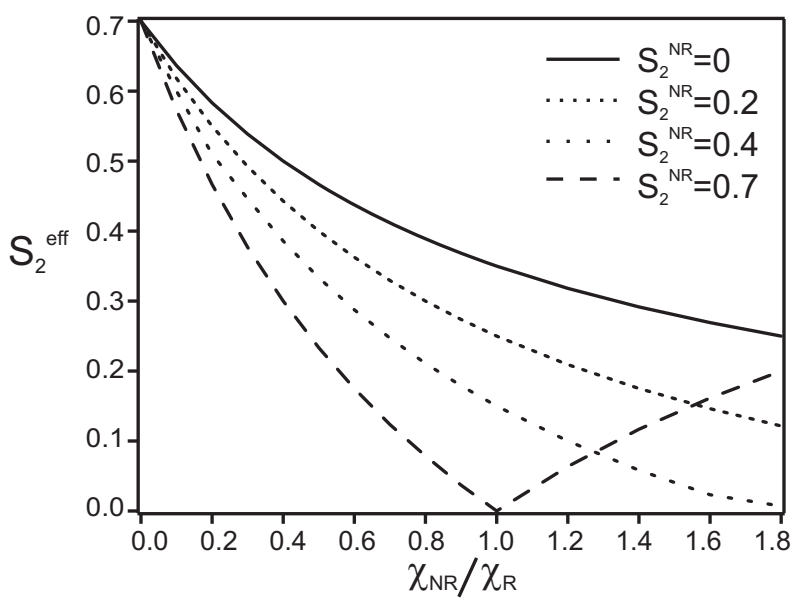

Figure 5: $S_{2}^{R}$ as function of the ratio between $\chi^{N R}$ and $\chi^{R}$ for different $S_{2}^{N R}$. The $\chi_{R}$ is made with $S_{2}^{R}=0.7, \varphi_{2}^{R}=0^{\circ}$ and $S_{4}^{(s)}=0$. The $\chi^{N R}$ is made with different values of $S_{2}^{N R}$ and with an orientation $\varphi_{2}^{N R}=90^{\circ}$

veloped to quantify the non resonant background impact on the measured $S_{2}$ values. While SRS is only sensitive to the imaginary part of the susceptibility, CARS is sensitive to the total susceptibility tensor $\chi$ :

$$
\chi=\chi^{N R}+\chi^{R}(\Omega)
$$

The total susceptibility read by the CARS process is then the susceptibility read by the SRS process plus an unknown non resonant contribution. If an isotropic non resonant background $\left(S_{2}^{N R}=0\right)$ is present, the polarization-resolved CARS intensity modulation is expected to decrease as a function of the magnitude of the non resonant part. Furthermore, if the non resonant background is itself organized $\left(S_{2}^{N R} \neq 0\right)$, the interpretation can be even more difficult. $^{27}$

In our simple model, a resonant susceptibility $\chi^{R}$ is assumed to originate from an anisotropic distribution of dipoles for which $S_{2}^{R}=0.7$ (as obtained from SRS measurements on MLVs in the CH2 symetric stretching bond region), and $S_{4}^{R}=0$. The corresponding $\chi^{R}$ susceptibility is calculated using Eq. 2. A non resonant susceptibility $\chi^{N R}$ is then added to this resonant part in order to build the whole susceptibility $\chi$ read-out by the CARS contrast. $\chi^{N R}$ is assumed to originate from various natures of symmetry orders, from totally isotropic 
$\left(S_{2}^{N R}=0\right)$ to highly organized $\left(S_{2}^{N R}=0.7\right)$, with $S_{4}^{N R}=0$. These non resonant second orders are moreover assumed to lie perpendicularly to the resonant contributions $\left(\varphi_{2}^{R} \perp \varphi_{2}^{N R}\right)$, consistent with the fact that non resonant signal comes mainly from electronic susceptibilities along the lipid tail, while the resonant $\mathrm{CH}_{2}$ stretching bond orients perpendicularly to this tail. ${ }^{7}$ From the resulting simulated polarization-dependent CARS intensity (Eq. 11), the "effective" $S_{2}^{e f f}$ values are deduced. These values are depicted in Fig. 5 for various strengths of non resonant with respect to resonant susceptibilities, and various symmetries of the non resonant background. Figure 5 shows that $S_{2}^{e f f}$ decreases with an increasing strength of the non resonant contribution, emphasizing the possible sources of bias induced by a non negligible non resonant background. In such case, the resulting read-out distribution, encompassing both resonant and non resonant contributions, can indeed become close to an apparent isotropic distribution $\left(S_{2}^{e f f}=0\right)$ in cases where $S_{2}^{R}=S_{2}^{N R}$.

Our experimental results described above have then been confronted to this theoretical model. The CARS non resonant background intensity is seen to be about $30 \%$ of the maximum resonant intensity, which corresponds roughly to $\chi^{N R} / \chi^{R} \approx \sqrt{0.3} \approx 0.55$. The $S_{2}^{N R}$ values obtained in the non resonant region are within the range [0.3-0.5] (Fig.4), which is in agreement with previously reported values in similar MLVs.${ }^{7}$ Similarly to what is assumed in the model described above, it has been furthermore experimentally reported that the non resonant part in lipids is dominated by the nonlinear susceptibility of carbon-carbon bonds. ${ }^{5}$ The mean direction of the $\mathrm{C}-\mathrm{C}$ bonds are oriented at a right angle of the $\mathrm{CH}_{2}$ bonds in MLV, supporting the assumption that $\varphi_{2}^{R}$ and $\varphi_{2}^{N R}$ are perpendicular. Following Fig. 5, this situation corresponds to a $S_{2}^{\text {eff }}$ which would lie around [0.3-0.4], which corresponds indeed to the experimental values obtained in Figs. 3 and 4. This analysis shows that molecular orientational order values measured in the context of polarized CARS imaging can be easily under-estimated due to the existence of a non negligible non resonant background. Measuring a reliable orientational information can be nevertheless obtained providing that the resonant strength is large enough with respect to the non resonant background. In the case 
of SRS, this issued does not occur providing that sources of artifact have been removed. ${ }^{22}$

\section{Conclusion}

A polarization-resolved method was performed in order to read out the parameters of molecular orientational organization using CRS microscopy. We found that SRS and CARS are not sensitive to the same degree of organization due to their intrinsic properties. SRS provides reliable information about the molecular bonds organization, whereas CARS can be biased by a non resonant contribution. In the case of lipids organization in MLV, the non resonant contribution is known and can be incorporated to the CARS analysis in order to explain the differences observed. More generally, the method described here to analyze polarization-resolved SRS and CARS intensity will allow new, fast and accurate observation of the molecular organization in biological samples.

$\dagger$ These authors contributed equally to this work.

\section{Acknowledgement}

The authors acknowledge finacial support from the Centre National de la Recherche Scientifique (CNRS), Aix Marseille University A*Midex (No. ANR-11-IDEX-0001-02). This work has been supported by Contracts ReceptORIENT (No. ANR-2010-BLAN-150902), FranceBioImaging (No. ANR-10-INBS-04-01), France Life Imaging (No. ANR-11-INSB-0006) and by the Conseil Regional Provence Alpes Cote dAzur Region. F.-Z.B. was supported by the ErasmusMundus Doctorate Program Europhotonics (Grant No. 159224-1-2009-1-FR-ERA MUNDUS-EMJD).

\section{References}

(1) Brasselet S. Polarization Resolved Nonlinear Microscopy: Application to Structural Molecular and Biological Imaging. Adv. Opt. Photon. 2011, 3, 205-271 
(2) Stoller P.; Kim B.-M.; Rubenchik A. M.; Reiser K. M.; Da Silva L. B. PolarizationDependent Optical Second-Harmonic Imaging of Rat-Tail Tendon. J. Biomed. Opt. 2002, 7, 205-214

(3) Gusachenko I.; Houssen Y. G.; Tran V.; Allain J.-M.; Schanne-Klein M.-C. PolarizationResolved Second-Harmonic Microscopy in Tendon Upon Mechanical Stretching. Biophys. J. 2012,102, 2220-2229

(4) Duboisset J.; Ait-Belkacem D.; Roche M.; Rigneault H.; Brasselet S. Generic Model of the Molecular Orientational Distribution Probed by Polarization Resolved Second Harmonic Generation. Phys. Rev. A 2012, 85, 043829

(5) Zimmerley M.; Mahou P.; Débarre D.; Schanne-Klein M.-C.; Beaurepaire E. Probing Ordered Lipid Assemblies with Polarized Third-Harmonic-Generation Microscopy. Phys. Rev. X 2013, 3, 011002

(6) Munhoz F.; Rigneault H.; Brasselet S. Polarization-Resolved Four-Wave Mixing for Structural Imaging in Thick Tissues. J. Opt. Soc. Am. B 2012, 29, 1541-1550

(7) Bioud F-Z.; Gasecka P.; Ferrand P.; Rigneault H.; Duboisset J.; Brasselet S. Structure of Molecular Packing Probed by Polarization-Resolved Nonlinear Four-Wave Mixing and Coherent Anti-Stokes Raman-Scattering Microscopy. Phys. Rev. A 2014, 89, 013836

(8) Cheng J. X.; Pautot S.; Weitz D. A.; Xie X. S.; Ordering of Water Molecules Between Phospholipid Bilayers Visualized by Coherent Anti-Stokes Raman Scattering Microscopy, Proc. Natl. Acad. Sci. 2003, 100, 9826-9830

(9) Zimmerley M.; Younger R.; Valenton T.; Oertel D. C.; Ward J. L.; Potma E. O. Molecular Orientation in Dry and Hydrated Cellulose Fibers: A Coherent Anti-Stokes Raman Scattering Microscopy Study. J. Phys. Chem. B 2010, 114, 10200-10208 
(10) Fu Y.; Huff T.B.; Wang H.-W.; Wang H.; Cheng J.-X. Ex Vivo and in Vivo Imaging of Myelin Fibers in Mouse Brain by Coherent Anti-Stokes Raman Scattering Microscopy. Opt. Express 2008, 16, 19396-19409

(11) Zumbusch A.; Holtom G. R.; Xie X. S. Three-Dimensional Vibrational Imaging by Coherent Anti-Stokes Raman Scattering. Phys. Rev. Lett. 1999, 82, 41424145

(12) Wang H.; Fu Y.; Zickmund P.; Shi R.; Cheng J.-X. Coherent Anti-Stokes Raman Scattering Imaging of Axonal Myelin in Live Spinal Tissues. Biophys. J. 2005, 89, $581-591$

(13) Bloembergen N. The Stimulated Raman Effect. A. J. Phys. 1967, 35, 989-1023

(14) Freudiger C. W.; Min W.; Saar B. G.; Lu S.; Holtom G. R.; He C.; Tsai J. C.; Kang J. X.; Xie X. S. Label-Free Biomedical Imaging with High Sensitivity by Stimulated Raman Scattering Microscopy. Science 2008, 19, 1857-1861

(15) Saar B. G.; Freudiger C. W.; Reichman J.; Stanley C. M.; Holtom G. R.; Xie X. S. Video-Rate Molecular Imaging in Vivo with Stimulated Raman Scattering. Science 2010, 3, 1368-1370

(16) Davis R. P.; Moad A. J.; Goeken G. S.; Wampler R. D.; Simpson G. J. Selection Rules and Symmetry Relation for Four-Wave Mixing Measurements of Uniaxial Assemblies, J. Phys. Chem. B 2008, 112, 5834-5848

(17) Munhoz F.; Brustlein S.; Hostein R.; Berto P.; Brasselet S.; Rigneault H. Polarization Resolved Stimulated Raman Scattering: Probing Depolarization Ratios of Liquids. J. Raman Spectrosc. 2012, 43, 419424

(18) De Vito G.; Bifone A.; Piazza V. Rotating-Polarization CARS microscopy: Combining Chemical and Molecular Orientation Sensitivity. Opt. Express 2012, 20, 29369-29377 
(19) Ferrand P.; Gasecka P.; Kress A.; Wang X.; Bioud F.-Z.; Duboisset J.; Brasselet S. Ultimate Use of Two-Photon Fluorescence Microscopy to Map Orientational Behavior of Fluorophores. Biophys. J. 2014, 106, 2330-2339

(20) Bangham A. D.; Hill M. W.; Miller N. G. A. Preparation and Use of Liposomes as Models of Biological Membranes. Methods in Membrane Biology 1974, 01, 1-68

(21) Brustlein S.; Ferrand P.; Walther N.; Brasselet S.; Billaudeau C.; Marguet D.; Rigneault H. Optical Parametric Oscillators Based Light Source for Coherent Raman Scattering Microscopy: a Practical Overview. J. Biomed. Opt. 2011, 16, 021106

(22) Berto P.; Andresen E. R.; Rigneault H. Background-Free Stimulated Raman Spectroscopy and Microscopy. Phys. Rev. Lett. 2014, 112, 053905

(23) Ferrand P. Scanning Imaging: a General Purpose Virtual Instrument for the Control of High-Speed Data Acquisition Devices. submitted

(24) Levin I. W.; Clark R. J. H.; Hester R. E. Advances in Infrared and Raman Spectroscopy. Eds. Wiley Heyden: New York, 1984, 11, 1-48

(25) Potma E. O.; Xie X. S. Detection of Single Lipid Bilayers with Coherent Anti-Stokes Raman Scattering (CARS) Microscopy, J. Raman Spectrosc. 2003, 34, 642-650

(26) Wurpel G. W.; Rinia H. A.; Muller M. Imaging Orientational Order and Lipid Density in Multilamellar Vesicles with Multiplex CARS Microscopy. J. Microsc. 2005, 218, 37-45

(27) Munhoz F.; Rigneault H.; Brasselet S. High Order Symmetry Structural Properties of Vibrational Resonances Using Multiple-Field Polarization Coherent Anti-Stokes Raman Spectroscopy Microscopy. Phys Rev Lett. 2010, 105, 123903

(28) Shang C. C.; Hsu H. The Spatial Symmetrical Forms of Third-Order Nonlinear Susceptibility, IEEE J. Quant. Electron. 1987, 23, 177-179 


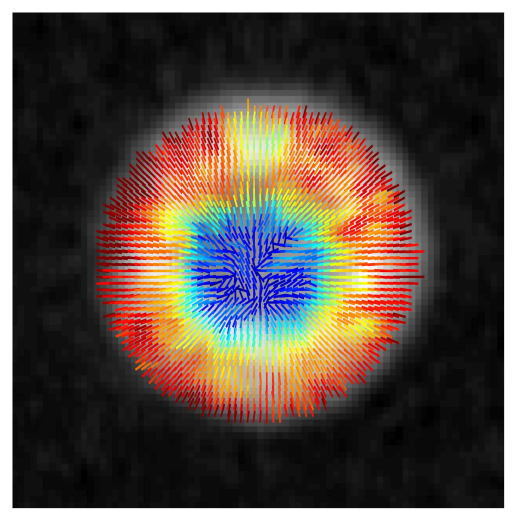

Figure 6: TOC graphic 